\title{
EFFECTS OF MULTIPATH AND CONVENTIONAL NMES ON MAXIMUM COMFORTABLE STIMULUS AND TORQUE PRODUCTION
}

\author{
Cody B. Bremner, ${ }^{1, A, B, C, D, E}$ William R. Holcomb ${ }^{2, A, B, D}$ \\ ${ }^{1}$ Department of Kinesiology \& Outdoor Recreation, Southern Utah University, USA \\ ${ }^{2}$ College of Health Professions, Mercer University, USA \\ ${ }^{\text {A }}$ Study Design; ${ }^{\mathrm{B}}$ Data Collection; ${ }^{\mathrm{C}}$ Statistical Analysis; ${ }^{\mathrm{D}}$ Manuscript Preparation; ${ }^{\mathrm{E}}$ Funds Collection \\ Address for correspondence: \\ Cody B. Bremner, PhD, LAT, ATC \\ 351 W. University Blvd., PEB 209 \\ Cedar City, UT 84720, USA \\ E-mail: codybremner@suu.edu
}

\begin{abstract}
Ahstract A novel multipath NMES (m-NMES) device has shown improved outcomes relative to conventional NMES (c-NMES) during recent basic and training studies. However, the mechanisms by which m-NMES outperformed c-NMES remain unclear. This study aimed to better understand these mechanisms by comparing the effects of m-NMES and c-NMES on maximum comfortable stimulus intensity and the subsequent NMES-induced torque, as these variables ultimately impact NMES training intensity; which is considered to be the primary determinant of NMES effectiveness.

We measured maximum comfortable stimulus intensity and the subsequent NMES-induced torque while participants performed NMES-induced contractions under two conditions (m-NMES and c-NMES).

Maximum comfortable stimulus intensity was significantly greater under the m-NMES condition, but the subsequent NMES-induced torque was not significantly different across conditions.

m-NMES does not appear to influence the outcomes in a clinically meaningful manner, since it performed similarly to c-NMES with respect to peak NMES-induced torque.
\end{abstract}

Key worlls multipath, NMES, quadriceps

\section{Introduction}

Neuromuscular electrical stimulation (NMES) treatments are common in orthopedic clinical settings as they can be used for a variety of purposes (Gondin, Cozzone, Bendahan, 2011; Holcomb, 1997; Lake, 1992). Despite this versatility, NMES is most often used for the specific goal of enhancing muscular strength. The effectiveness of NMES for this purpose is believed to be primarily determined by NMES training intensity (Maffiuletti, 2010; Maffiuletti, Minetto, Farina, Bottinelli, 2011), which is often defined as the ratio of NMES-induced torque to torque produced during a maximum voluntary isometric contraction (expressed as \% MVIC) (Gondin et al., 2011). Accordingly, clinicians are encouraged to maximize NMES training intensities to the degree possible (Maffiuletti, 
2010), but the ability to achieve and maintain appropriate NMES training intensities is limited by a variety of factors; which include: patient discomfort (Gobbo, Maffiuletti, Orizio, Minetto, 2014; Gondin et al., 2011; Maffiuletti, 2010), muscle fatigue (Doucet, Lam, Griffin, 2012; Laufer, Elboim, 2008; Maffiuletti, 2010; Maffiuletti, Vivodtzev, Minetto, Place, 2014) and spatially limited motor unit recruitment (Gobbo et al., 2014; Maffiuletti, 2010; Maffiuletti et al., 2014).

The Kneehab ${ }^{2}$ XP (Theragen LLC, Leesburg, VA) is an electrical stimulator that has received substantial attention in the literature (Asakawa, Jung, Koh, 2014; Bremner Holcomb, In-press; Bruce-Brand et al., 2012; Coote, Hughes, Rainsford, Minogue, Donnelly, 2015; Feil, Newell, Minogue, Paessler, 2011; Maffiuletti et al., 2014; Morf, Wellauer, Casartelli, Maffiuletti, 2015; Paessler, 2012; Walls, McHugh, O'Gorman, Moyna, O'Byrne, 2010), because it implements a novel strategy designed to address the aforementioned primary factors limiting NMES training

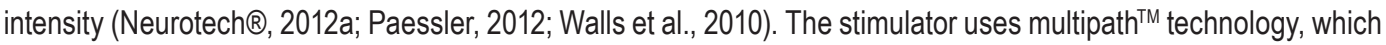
distributes the electrical current between four large electrodes integrated within a neoprene thigh garment via two separate channels while also altering pulse durations (Maffiuletti et al., 2014; Morf et al., 2015; Paessler, 2012; Walls et al., 2010); thus it is referred to as multipath NMES (m-NMES). In contrast, conventional NMES (c-NMES) stimulators distribute the electrical current in each channel via a single fixed path between a pair of electrodes. It has been suggested that m-NMES is advantageous because it provides an asynchronous stimulus and improves spatial distribution through dynamically changing the pathways by which current is distributed and by dynamically altering the pulse duration (Feil et al., 2011; Gobbo et al., 2014). For example, greater spatial distribution of the NMES stimulus may results in greater torque production, as improved spatial distribution may maximize the number of motor units recruited by the stimulus (Maffiuletti et al., 2014).

To date, a single randomized controlled trial comparing the use of m-NMES and c-NMES on clinical outcomes has been completed, with S. Feil et al. (2011) observing greater improvements following ACL reconstruction while using the m-NMES device. However, the authors acknowledged that the mechanism(s) responsible for their observations remain unclear. Subsequent basic studies have attempted to identify the possible mechanism(s) by which m-NMES outperformed c-NMES, with mixed results. Two studies comparing m-NMES and c-NMES observed improved fatigue and discomfort related outcomes while using m-NMES (Maffiuletti et al., 2014; Morf et al., 2015), but substantially different electrode configurations were used across conditions; thus the authors' ability to attribute their observations to the novel multipath current distribution method was limited. Consequently, a similar basic study was performed in our laboratory while using similar electrode configurations because this approach allowed us to better examine the influence of the novel multipath current distribution method on these outcomes (Bremner, Holcomb, in-press). Using this approach, we did not observe any clinically relevant differences across the two conditions.

We standardized the NMES stimulus intensity across conditions during our previous study to limit baseline differences, which is necessary when comparing fatigue and discomfort related outcomes. However, this methodology did not allow us to examine differences in maximum comfortable stimulus intensity and the subsequent NMES-induced torque; which are also important outcomes when comparing NMES treatment conditions because they impact NMES training intensity (Bremner, Holcomb, Brown, 2015; Dantas, Vieira, Siqueira, Salvini, Durigan, 2015; Holcomb, Golestani, Hill, 2000). To the best of our knowledge, the influence of m-NMES on maximum comfortable stimulus intensity and the subsequent NMES-induced torque while using similar electrode configurations has yet to be examined. Each of these outcome measures are clinically relevant and warrant further investigation, as they ultimately impact the NMES training intensity. Therefore, the purpose of this study is to compare the effects 
of m-NMES and c-NMES on the clinically relevant maximum comfortable stimulus intensity and subsequent NMES-induced torque outcomes. We hypothesize that the maximum comfortable stimulus intensity and subsequent NMES-induced torque will be greater while using m-NMES.

\section{Methods \\ Design}

We performed a single-blind counterbalanced crossover study with 1 independent variable (NMES condition at 2 levels: m-NMES and c-NMES) and 2 dependent variables (maximum comfortable stimulus intensity and NMESinduced torque). We assigned participants to one of two permutations designed to counterbalance the session order in which the c-NMES and m-NMES treatment conditions were performed.

\section{Participants}

We performed an a priori power analysis using G*Power software (version 3.1.9.2) to determine a target sample size (Faul, Erdfelder, Lang, Buchner, 2007). We determined a target sample size of 17 participants in order to maintain adequate power $(1-\beta=0.80)$ and detect a medium to large effect size $(d=0.650)$ while using a dependent $t$-test (Cohen, 1988). We selected medium to large effect sizes for the power analysis because we believe that any statistically significant differences with corresponding effect sizes smaller than this threshold would lack clinical relevance for the outcomes included in our study.

A convenience sample of 21 participants (age $=23.9 \pm 5.1$ years, height $=175.1 \pm 7.4 \mathrm{~cm}$, mass $=78.1$ $\left.\pm 11.7 \mathrm{~kg}, \mathrm{BMI}=25.3 \pm 2.6 \mathrm{~kg} / \mathrm{m}^{2}\right)$ from the university and community completed two study sessions. As has been done previously (Gorgey, Dudley, 2008), participants in our current study had prior NMES experience due to their participation in an earlier study (Bremner Holcomb, In-press). We elected to use participants from a previous study because an individual's tolerance to NMES is likely to improve over the first few exposures to NMES treatments (Alon, Smith, 2005).

Participants were required to be healthy, recreationally active, males, between the ages of $18-35$. Participants also had to have a body mass index $(\mathrm{BMI}) \leq 30 \mathrm{~kg} / \mathrm{m}^{2}$ to be included, as NMES tolerance and motor thresholds have been shown to differ between individuals with a BMI above and below $30 \mathrm{~kg} / \mathrm{m}^{2}$ (Maffiuletti, Morelli, et al., 2011). To be included in our current study participants had to tolerate a NMES training intensity of at least $30 \%$ MVIC during a previous study. This study was approved by the University's institutional review board and participants provided written informed consent. To facilitate participant recruitment, we incentivized participants via a lottery for a chance to win one of four $\$ 50$ gift cards.

\section{Instrumentation}

We used a Quickset 4 Biodex dynamometer (Biodex Medical Systems Inc., Shirley, New York) to measure and record isometric knee extension torque following procedures used previously (Bremner, Holcomb, In-press). We applied the c-NMES treatment using the same Sonicator ${ }^{\circledR}$ Plus 940 stimulator (Mettler Electronics ${ }^{\circledR}$ Corp., Anaheim, CA). To maintain consistency across the two NMES conditions, we set the c-NMES parameters as similar as possible to the parameters used with the Kneehab® XP program 6 (Table 1). We used four self-adherent electrodes to deliver the c-NMES current (two $-5 \mathrm{~cm} \times 9 \mathrm{~cm}$ [Metron ${ }^{\top \mathrm{M}}$, Bolingbrook, IL], one $-10.79 \mathrm{~cm} \times 17.78 \mathrm{~cm}$ 
[TENS Products, Grand Lake, CO], one $-7 \mathrm{~cm} \times 14 \mathrm{~cm}$ electrode (SME INC., Wilmington, NC; Figure 1). To guide the placement of the c-NMES electrodes, we manually identified four motor points that would allow us to place the c-NMES electrodes in a similar fashion to the m-NMES electrode configuration (proximal and distal vastus lateralis, proximal rectus femoris and distal vastus medialis) using a pencil electrode (Mettler Electronics XK2, Active Forever, Scottsdale, AZ; Figure 2) (Gobbo et al., 2014).

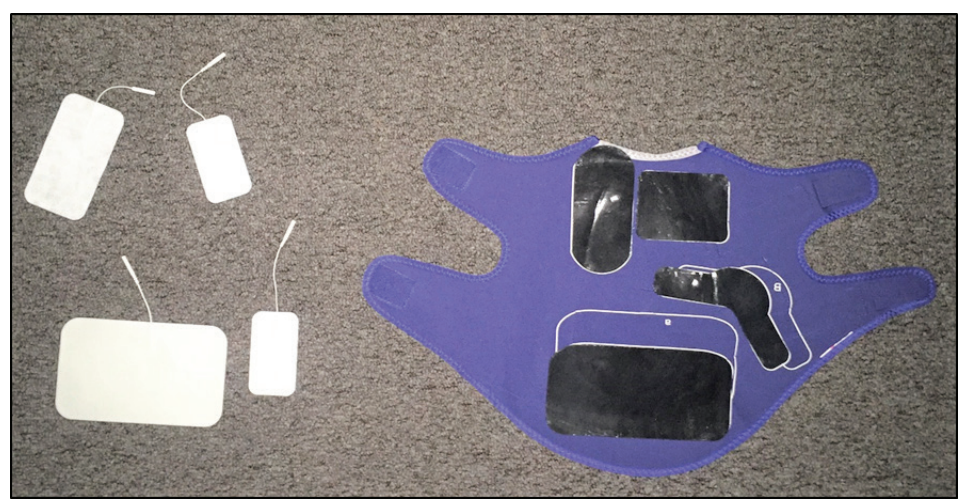

Note: c-NMES electrodes are on the left side of the photo and m-NMES electrodes are integrated into the neoprene garment on the right side of the photo.

Figure 1. Electrode Configuration Comparison

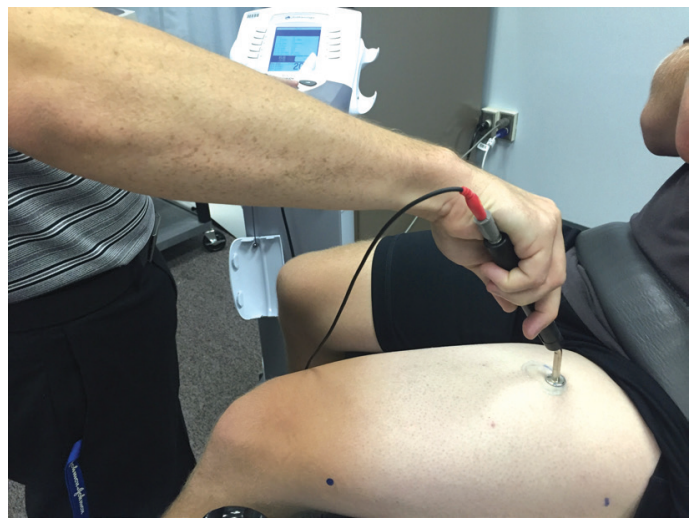

Note: the photo illustrates the pencil electrode method for manually identifying motor points.

Figure 2. Motor Point Identification

We applied the m-NMES treatment using the same Kneehab® XP stimulator (Theragen LLC, Leesburg, VA), however we assigned each participant a separate Kneehab® XP garment with integrated electrodes. We integrated the m-NMES electrodes into the neoprene garment and subsequently placed the garment on the dominant thigh 
according to the manufacturer's recommendations (Figure 3) (Neurotech®, 2012b). We set the stimulator parameters to program 6 during all m-NMES treatments (Table 1).

Table 1. Parameters of Neuromuscular Electrical Stimulation Conditions

\begin{tabular}{|c|c|c|}
\hline Parameter & m-NMES & c-NMES \\
\hline Current distribution & Multipath & Single path within two independent channels \\
\hline Waveform & Biphasic Square & Biphasic Square \\
\hline Frequency & $70 \mathrm{~Hz}$ & $70 \mathrm{~Hz}$ \\
\hline Pulse duration & $400 / 100 \mu \mathrm{sec}$ & $400 \mu \mathrm{sec}$ \\
\hline Ramp & 1 second up : 0.5 seconds down & 1 second up : 0 seconds down ${ }^{*}$ \\
\hline On time/off time & $10 \mathrm{~s} / 50 \mathrm{~s}$ & $10 \mathrm{~s} / 50 \mathrm{~s}$ \\
\hline Stimulus intensity & Maximum comfortable & Maximum comfortable \\
\hline Number of electrodes & 4 & 4 \\
\hline Total area of electrodes & $427 \mathrm{~cm}^{2}$ & $360 \mathrm{~cm}^{2 *}$ \\
\hline
\end{tabular}

* It was not possible to select a ramp-down of 0.5 seconds with this particular c-NMES device while also maintaining a similar ramp-up and hold time to the m-NMES device, thus a ramp-down was not included. A slightly smaller total area of electrodes was used during the c-NMES condition.

\section{Procedures}

Participants reported at the same time of day ( \pm 2 hours) on two occasions and each session lasted approximately 1 hour. Each participant's dominant leg, which was defined as the leg with which they would use to kick a soccer ball, served as the leg of interest throughout the study (20 right, 1 left). We also instructed participants to report well hydrated and to refrain from strenuous activities for 12 hours prior to reporting.

Each session began with the participants completing a standardized warm-up following procedures used previously (Bremner, Holcomb, in-press). Participants rested for 8 minutes following the warm-up, during which we identified the motor points using the pencil electrode method and cleaned the leg of interest with an alcohol free wipe. Although motor point identification was not necessary for the m-NMES condition because the electrodes were integrated within the garment, we still identified motor points during both sessions in an effort to blind participants to treatment condition.

To continue the warm-up, participants performed maximum voluntary isometric contractions (MVICs) of the quadriceps for 6 seconds in duration and then rested for 5 minutes prior to performing the NMES procedures, during which we placed the Kneehab® XP garment with integrated electrodes or the c-NMES electrodes over the participant's shaved dominant thigh. We also placed an empty Kneehab® XP garment over the c-NMES electrodes in an effort to blind participants to treatment condition (Figure 3) (Morf et al., 2015). As has been done previously to limit fatigue (Bremner et al., 2015), participants performed a single NMES-induced contraction during each session (c-NMES or m-NMES) while using a self-selected maximum comfortable stimulus intensity; which is defined as the highest intensity that does not cause pain (Holcomb, Rubley, Girouard, 2007).The maximum comfortable stimulus intensity was determined following procedures used previously (Figure 3) (Bremner, Holcomb, in-press). 

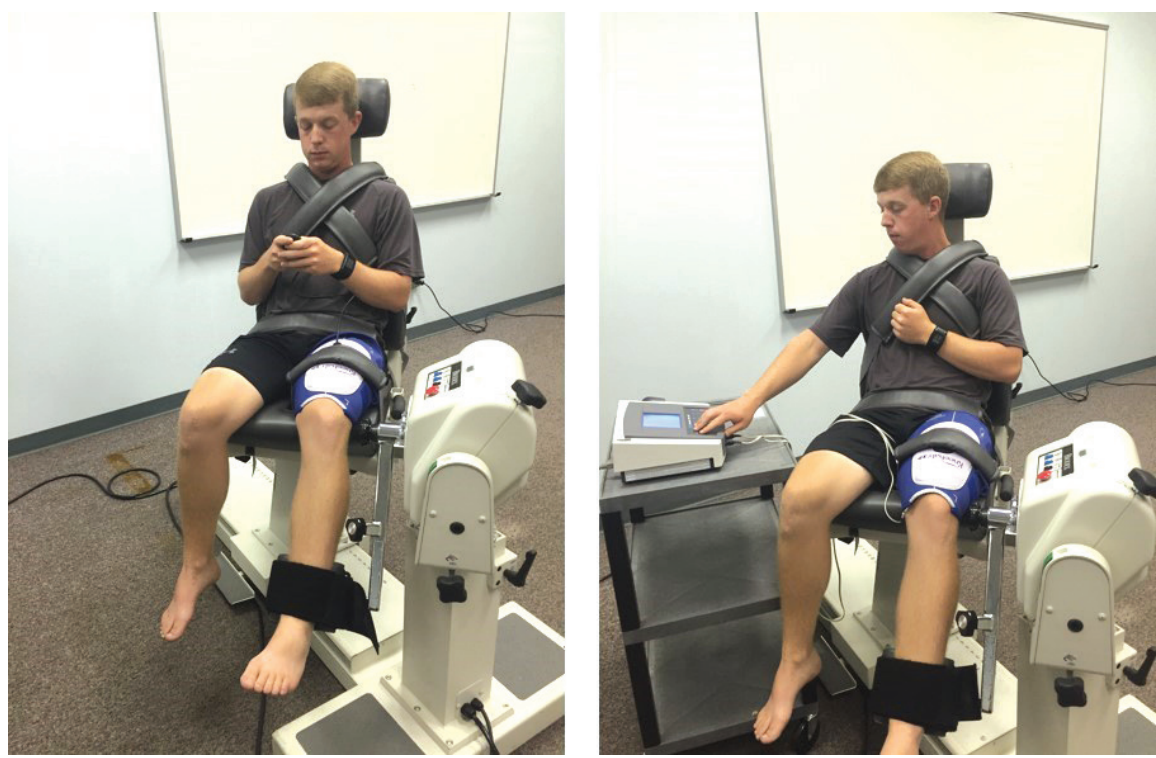

Note: the photo on the left illustrates the participant self-selecting a maximum comfortable stimulus intensity with the m-NMES device. The photo on the right illustrates the participant self-selecting a maximum comfortable stimulus intensity with the c-NMES device.

Figure 3. NMES Treatments

\section{Outcome Measures}

Maximum Comfortable Stimulus Intensity. We manually recorded the maximum comfortable stimulus intensity selected by each participant (expressed in milliamps [mA]). The m-NMES device does not express the stimulus intensity in $\mathrm{mA}$ units, thus a conversion table provided by the manufacturer was used to convert the observed m-NMES stimulus intensities into the appropriate units.

Normalized NMES-induced Torque. The isokinetic dynamometer measured and recorded the NMES-induced peak torque under each condition. In an effort to reduce inter-participant variability, we normalized the NMES-induced peak torque values to each participant's body mass, which converts the unit of measure to Newtonmeters per kilogram (Nm/kg) and has been done previously (Bremner et al., 2015; Holcomb et al., 2000).

\section{Statistical Analysis}

We used the Statistical Package for Social Sciences (SPSS) version 23.0 (IBM Corporation, Armonk, NY) to analyze the data. We performed a separate dependent $t$-test to analyze each outcome measure. To examine the magnitude of the differences, we calculated Cohen's $d$ effect sizes (Cohen, 1988). We calculated Cohen's $d$ effect sizes corresponding to within groups comparisons using the equation suggested by Cumming (2012) which uses the average standard deviation of the paired data as the standardizer $\left(\mathrm{d}_{\text {sav }}\right)$. Since $d$ statistics are believed to overestimate the population effect size, Cumming recommended that an unbiased Cohen's $d\left(d_{\text {unb }}\right)$ also be provided. Accordingly, we calculated $d_{u n b}$ values using the equation provided by Cumming. 


\section{Resullts}

Prior to analyzing the data, we assessed the tenability of the applicable statistical assumptions, and the data were considered to be normally distributed without any outliers.

\section{Maximum Comfortable Stimulus Intensity}

The maximum comfortable stimulus intensity $(\mathrm{mA})$ was significantly higher during the m-NMES condition $\left(t_{20}=2.817 ; P=0.006 ; d=0.581 ; 95 \% \mathrm{Cl}\right.$ for effect size: $-0.133,1.018 ; d_{u n b}=0.559 ;$ Figure 4$)$.

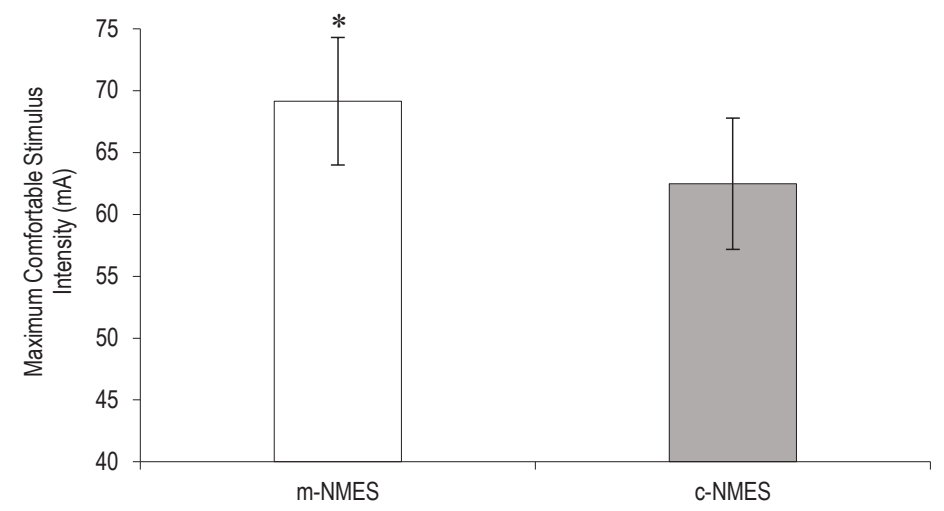

Note: * Significantly greater maximum comfortable stimulus intensity $(P=0.006)$. Error bars indicate $95 \%$ confidence intervals calculated using a critical $t$-value as has been recommended (Cumming, 2012).

Figure 4. Maximum Comfortable Stimulus Intensity

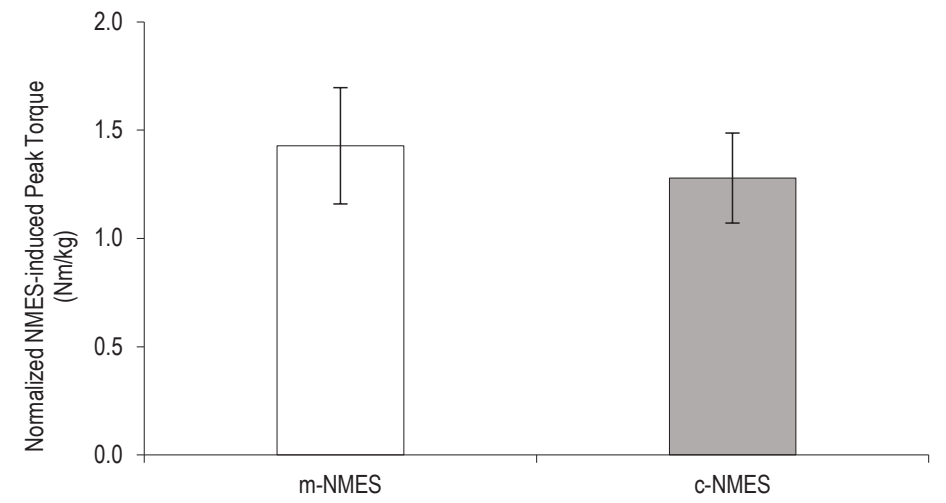

Note: error bars indicate $95 \%$ confidence intervals and were calculated using a critical $t$-value as has been recommended (Cumming, 2012).

Figure 5. Initial Normalized NMES-induced Peak Torque 


\section{Normalized NMES-induced Torque}

The normalized NMES-induced torque $(\mathrm{Nm} / \mathrm{kg})$ was not significantly different across conditions $\left(\mathrm{t}_{20}=1.397\right.$; $P=0.089 ; d=0.282 ; 95 \% \mathrm{Cl}$ for effect size: $-0.125,0.683 ; d_{u n b}=0.272 ;$ Figure 5$)$.

\section{Discussion}

While using similar electrode configurations, the findings of our study indicate that the maximum comfortable stimulus intensity was significantly higher under the m-NMES condition. However, the higher stimulus intensity did not result in significantly greater NMES-induced torque production during the subsequent NMES-induced contraction. Due to the positive linear relationship between stimulus intensity and NMES-induced torque (Adams, Harris, Woodard, Dudley, 1993; Gorgey, Mahoney, Kendall, Dudley, 2006; Maffiuletti, 2010), the primary clinical objective of using higher stimulus intensities is to enhance the NMES training intensity by increasing NMES-induced torque production. Therefore, the greater maximum comfortable stimulus intensity that we observed during the m-NMES condition does not appear to be clinically meaningful.

Despite our efforts to standardize the electrode configurations, the m-NMES electrodes covered an area of $427 \mathrm{~cm}^{2}$ while the c-NMES electrodes covered a surface area of roughly $360 \mathrm{~cm}^{2}$ (Morf et al., 2015). Since the current was spread over a greater area during the m-NMES condition, the current density $\left(\mathrm{mA} / \mathrm{cm}^{2}\right)$ was subsequently lower during this condition while using the same amount of current (Hooker, 2003). We observed similar values when normalizing the mean stimulus intensities by total electrode area for each condition (m-NMES $\left.=0.16 \mathrm{~mA} / \mathrm{cm}^{2}, \mathrm{c}-\mathrm{NMES}=0.17 \mathrm{~mA} / \mathrm{cm}^{2}\right)$. Therefore, the difference in electrode sizes is a possible explanation as to why we did not observe significantly greater NMES-induced torque during the m-NMES condition. Although a small difference in the area covered by c-NMES and m-NMES remained during our study (Figure 1), the c-NMES electrode configurations used during previous studies consisted of three electrodes covering only $100 \mathrm{~cm}^{2}$ (Maffiuletti et al., 2014; Morf et al., 2015).

The maximum comfortable stimulus intensities we observed under the m-NMES and c-NMES conditions were $69.1 \pm 11.3 \mathrm{~mA}$ and $62.5 \pm 11.6 \mathrm{~mA}$, respectively (Figure 4). During a similar study Maffiuletti et al. (2014) reported values of $92 \pm 25 \mathrm{~mA}$ and $53 \pm 25 \mathrm{~mA}$ during their m-NMES and c-NMES conditions. Despite the fact that both of these studies observed significantly greater stimulus intensities under the m-NMES condition, the mean stimulus intensity we observed during m-NMES is much smaller. Two likely explanations for this difference are that Maffiuletti et al. used a maximum tolerable stimulus intensity and a modified m-NMES device that allowed a maximum current output of $200 \mathrm{~mA}$. We elected to use a lower threshold maximum comfortable stimulus intensity because it has been suggested to be more clinically relevant (Holcomb et al., 2007). In addition, Maffiuletti et al. acknowledged that their use of a modified research version of the m-NMES device was a limitation of their study, as it is not available to clinicians, so we elected to use the clinically available m-NMES device with a maximum output of only $79.2 \mathrm{~mA}$.

During the m-NMES and c-NMES conditions we observed normalized NMES-induced torque values of 1.4 $\pm 0.6 \mathrm{Nm} / \mathrm{kg}$ and $1.3 \pm 0.5 \mathrm{Nm} / \mathrm{kg}$, respectively (Figure 5). It is difficult for us to directly compare these values to similar studies comparing m-NMES and c-NMES because normalized torque values were not reported (Maffiuletti et al., 2014; Morf et al., 2015). To facilitate the comparison of our results to these previous studies, we converted the normalized NMES-induced torque values to NMES training intensities using the values recorded during the warm-up MVICs. The subsequent training intensities were $47.9 \pm 17.1 \%$ MVIC and $43.6 \pm 13.8 \%$ MVIC for m-NMES and c-NMES, 
respectively. Interestingly, our observed values are near the upper margin of the proposed therapeutic window of 25$50 \%$ MVIC (Alon, Smith, 2005). This observation suggests that both devices are capable of producing the torque required for effective NMES treatments, which may be of interest to clinicians as the m-NMES device is portable.

Although the previous studies comparing torque output across m-NMES and c-NMES conditions used a higher threshold maximum tolerable stimulus intensity (Maffiuletti et al., 2014; Morf et al., 2015), the NMES training intensities we observed are comparable to values reported during these studies; which ranged from roughly $35-45 \%$ MVIC. Despite using a lower threshold maximum comfortable stimulus intensity, we believe that the comparable NMES training intensities observed during our study are due to the participants' previous NMES experience, as this likely allowed participants to better acclimate to the NMES stimulus prior to participation in our current study (Alon, Smith, 2005). In contrast, one of the other studies comparing m-NMES and c-NMES did not incorporate familiarization sessions and the other included a single familiarization session (Maffiuletti et al., 2014; Morf et al., 2015).

Although we observed a significantly greater maximum comfortable stimulus intensity under the m-NMES condition, we did not observe a significant difference with respect to the NMES-induced torque across the two conditions. This observation is contrary to the results of previous studies (Maffiuletti et al., 2014; Morf et al., 2015), and methodological differences between our study and the previous studies warrant further discussion. Maffiuletti et al. and Morf et al. hypothesized that a possible mechanism for the significantly greater NMES-induced torque they observed during m-NMES was the novel multipath current distribution method. Maffiuletti et al. suggested that relative to the fixed single path current distribution method of c-NMES, a larger number of motor units may have been recruited during the m-NMES condition due to its greater spatial distribution of the stimulus. However, the m-NMES and c-NMES conditions during these studies differed in two systematic ways, which were the current distribution method and electrode configuration. Morf et al. indicated that as a result of these two systematic differences, it is unclear whether the greater NMES-induced torque they observed was primarily attributable to the multipath current distribution method, larger electrodes or a combination of these factors. Consequently, we standardized the electrode configuration across conditions to the extent possible during our study, as we believe this approach allowed us to better isolate the influence of current distribution method on NMES-induced torque. Since we did not observe significantly greater NMES-induced torque under the m-NMES condition, our results do not support the hypothesis of Maffiuletti et al. and Morf et al. that the multipath current distribution method is a possible mechanism by which m-NMES resulted in greater NMES-induced torque during their studies.

\section{Limitations}

Eight participants reached the output capacity of the m-NMES device prior to achieving their maximum comfortable threshold during our study. This likely prevented these participants from reaching their true maximum comfortable stimulus intensity during the m-NMES condition, and this may have subsequently reduced the magnitude of our observed effect. Although incorporating the clinically available device during our study may be viewed as a limitation, we feel that it ultimately enhances the clinical applicability of our findings; as the device used in previous studies is a modified version allowing $200 \mathrm{~mA}$, but is not available to clinicians.

The extent to which our results are generalizable is unclear, due to our use of healthy participants and exclusion of females. The menstrual cycle has been shown to influence self-reported discomfort levels (Teepker, Peters, Vedder, Schepelmann, Lautenbacher, 2010), thus due to our study design requiring repeated measurements over time we felt it was necessary to exclude females. In addition, during exploratory NMES studies, similar in nature to our study, it is 
common practice to use healthy participants (Alon, Smith, 2005; Dantas et al., 2015; Gorgey, Dudley, 2008; Holcomb et al., 2007; Holcomb, Rubley, Miller, Girouard, 2006; Holcomb, Rubley, Randolph, 2011; Maffiuletti et al., 2014).

\section{Conclusions}

The results of our study do not indicate that the novel multipath current distribution method improves the outcomes included in our study in a clinically meaningful manner. Contrary to our results, similar previous studies have observed improved outcomes when comparing m-NMES and c-NMES (Maffiuletti et al., 2014; Morf et al., 2015). We believe it is likely that contributing factors for their improved outcomes were differences in electrode configuration and their use of a modified version of the device not available to clinicians; rather than the novel current distribution method.

\section{Acknowledgments}

We thank Dr. Trenton E. Gould, Dr. Scott G. Piland and Dr. Gary V. Krebs for their assistance throughout this study. In addition, we thank Theragen LLC for providing the materials used in this study to perform the multipath neuromuscular electrical stimulation (m-NMES) condition.

\section{References}

Adams, G.R., Harris, R.T., Woodard, D., Dudley, G.A. (1993). Mapping of electrical muscle stimulation using MRI. Journal of applied physiology, 74 (2), 532-537.

Alon, G., Smith, G.V. (2005). Tolerance and conditioning to neuro-muscular electrical stimulation within and between sessions and gender. J Sports Sci Med, 4 (4), 395-405.

Asakawa, Y., Jung, J., Koh, S. (2014). Neuromuscular electrical stimulation improves strength, pain and weight distribution on patients with knee instability post surgery. Physical Therapy Rehabilitation Science, 3 (2), 112-118. DOI: 10.14474/ptrs.2014.3.2.112.

Bremner, C.B., Holcomb, W.R. (in-press). A Comparison of Multipath and Conventional Neuromuscular Electrical Stimulation. Athletic Training \& Sports Health Care.

Bremner, C.B., Holcomb, W.R., Brown, C.D. (2015). Knee Joint Angle Influences Neuromuscular Electrical Stimulation-Induced Torque. Athletic Training \& Sports Health Care, 7 (4), 165-172. DOI: 10.3928/19425864-20150707-07.

Bruce-Brand, R.A., Walls, R.J., Ong, J.C., Emerson, B.S., O'Byrne, J.M., Moyna, N.M. (2012). Effects of home-based resistance training and neuromuscular electrical stimulation in knee osteoarthritis: a randomized controlled trial. BMC musculoskeletal disorders, 13 (118), 1-10.

Cohen, J. (1988). Statistical Power Analysis for the Behavioral Sciences (2nd ed.). Hillsdale, N.J.: Lawrence Erlbaum Associates.

Coote, S., Hughes, L., Rainsford, G., Minogue, C., Donnelly, A. (2015). Pilot randomized trial of progressive resistance exercise augmented by neuromuscular electrical stimulation for people with multiple sclerosis who use walking aids. Arch Phys Med Rehabil, 96 (2), 197-204. DOI: 10.1016/j.apmr.2014.09.021.

Cumming, G. (2012). Understanding the New Statistics: Effect Sizes, Confidence Intervals, and Meta-analysis. New York, NY: Routledge.

Dantas, L.O., Vieira, A., Siqueira, A.L., Jr., Salvini, T.F., Durigan, J.L. (2015). Comparison between the effects of 4 different electrical stimulation current waveforms on isometric knee extension torque and perceived discomfort in healthy women. Muscle Nerve, 51 (1), 76-82. DOI: 10.1002/mus.24280.

Doucet, B.M., Lam, A., Griffin, L. (2012). Neuromuscular Electrical Stimulation for Skeletal Muscle Funciton. Yale Journal of Biology and Medicine, 85 (2), 201-215.

Faul, F., Erdfelder, E., Lang, A.G., Buchner, A. (2007). G*Power 3: a flexible statistical power analysis program for the social, behavioral, and biomedical sciences. Behav Res Methods, 39 (2), 175-191.

Feil, S., Newell, J., Minogue, C., Paessler, H.H. (2011). The effectiveness of supplementing a standard rehabilitation program with superimposed neuromuscular electrical stimulation after anterior cruciate ligament reconstruction: a prospective, randomized, single-blind study. Am J Sports Med, 39 (6), 1238-1247. DOl: 10.1177/0363546510396180. 
Gobbo, M., Maffiuletti, N.A., Orizio, C., Minetto, M.A. (2014). Muscle motor point identification is essential for optimizing neuromuscular electrical stimulation use. Journal of Neuroengineering and Rehabilitation, 11 (17), 1-6. DOI: 10.1186/1743-0003-11-17.

Gondin, J., Cozzone, P.J., Bendahan, D. (2011). Is high-frequency neuromuscular electrical stimulation a suitable tool for muscle performance improvement in both healthy humans and athletes? Eur J Appl Physiol, 111 (10), 2473-2487. DOI: 10.1007/ s00421-011-2101-2.

Gorgey, A.S., Dudley, G.A. (2008). The role of pulse duration and stimulation duration in maximizing the normalized torque during neuromuscular electrical stimulation. J Orthop Sports Phys Ther, 38 (8), 508-516. DOI: 10.2519/jospt.2008.2734.

Gorgey, A.S., Mahoney, E., Kendall, T., Dudley, G.A. (2006). Effects of neuromuscular electrical stimulation parameters on specific tension. Eur J Appl Physiol, 97 (6), 737-744. DOI: 10.1007/s00421-006-0232-7.

Holcomb, W.R. (1997). A practical guide to electrical therapy. Journal of Sport Rehabilitation, 6 (3), $272-282$.

Holcomb, W.R., Golestani, S., Hill, S. (2000). A comparison of knee-extension torque production with biphasic versus russian current. Journal of Sport Rehabilitation, 9, 229-239.

Holcomb, W.R., Rubley, M.D., Girouard, T.J. (2007). Effect of the Simultaneous Application of NMES and HVPC on Knee Extension Torque. Journal of Sport Rehabilitation, 16, 307-318.

Holcomb, W.R., Rubley, M.D., Miller, M.G., Girouard, T.J. (2006). The effect of rest intervals on knee-extension torque production with neuromuscular electrical stimulation. Journal of Sport Rehabilitation, 15 (2), 116-124.

Holcomb, W.R., Rubley, M.D., Randolph, S.M. (2011). Increasing Neuromuscular Electrical Stimulation Amplitude to Reduce the Decline in Knee Extension Torque. Athletic Training \& Sports Health Care, 3 (2), 63-68.

Hooker, D.N. (2003). Electrical Stimulating Currents. In: W.E. Prentice (ed.), Therapeutic Modalities for Sports Medicine and Athletic Training (5th ed., pp. 191-239). New York, NY: McGraw-Hill.

Lake, D.A. (1992). Neuromuscular electrical stimulation: an overview and its application in the treatment of sports injuries. Sports medicine, 13 (5), 320-336.

Laufer, Y., Elboim, M. (2008). Effect of burst frequency and duration of kilohertz-frequency alternating currents and of low-frequency pulsed currents on strength of contraciton, muscle fatigue, and percieved discomfort. Physical Therapy, 88 (10), 1167-1176.

Maffiuletti, N.A. (2010). Physiological and methodological considerations for the use of neuromuscular electrical stimulation. Eur J Appl Physiol, 110(2), 223-234. doi: 10.1007/s00421-010-1502-y

Maffiuletti, N.A., Minetto, M.A., Farina, D., Bottinelli, R. (2011). Electrical stimulation for neuromuscular testing and training: state-of-the art and unresolved issues. Eur J Appl Physiol, 111 (10), 2391-2397. DOI: 10.1007/s00421-011-2133-7.

Maffiuletti, N.A., Morelli, A., Martin, A., Duclay, J., Billot, M., Jubeau, M., ..., Sartorio, A. (2011). Effect of gender and obesity on electrical current thresholds. Muscle \& Nerve, 44 (2), 202-207.

Maffiuletti, N.A., Vivodtzev, I., Minetto, M.A., Place, N. (2014). A new paradigm of neuromuscular electrical stimulation for the quadriceps femoris muscle. Eur J Appl Physiol, 114 (6), 1197-1205. DOI: 10.1007/s00421-014-2849-2.

Morf, C., Wellauer, V., Casartelli, N.C., Maffiuletti, N.A. (2015). Acute effects of multipath electrical stimulation in patients with total knee arthroplasty. Arch Phys Med Rehabil, 96 (3), 498-504. DOI: 10.1016/j.apmr.2014.10.011.

Neurotech ${ }^{2}$ (2012a). Kneehab® XP Quadriceps Therapy System. Hoboken, NJ: Bio-medical research Ltd.

Neurotech® (2012b). Quick start guide for clinicians. Hoboken, NJ: Biomedical Research Ltd.

Paessler, H.H. (2012). Emerging Techniques in Orthopedics: Advances in Neuromuscular Electrical Stimulation. American Journal of Orthopedics, 41 (5 Suppl.), 1-8.

Teepker, M., Peters, M., Vedder, H., Schepelmann, K., Lautenbacher, S. (2010). Menstrual variation in experimental pain: correlation with gonadal hormones. Neuropsychobiology, 61 (3), 131-140.

Walls, R.J., McHugh, G., O'Gorman, D.J., Moyna, N.M., O'Byrne, J.M. (2010). Effects of preoperative neuromuscular electrical stimulation on quadriceps strength and functional recovery in total knee arthroplasty. A pilot study. BMC Musculoskelet Disord, 11 (119), 1-9. DOI: 10.1186/1471-2474-11-119.

Cite this anticle aS: Bremner, C.B., Holcomb, W.R. (2019). Effects of Multipath and Conventional NMES on Maximum Comfortable Stimulus and Torque Production. Central European Journal of Sport Sciences and Medicine, 1 (25), 23-33. DOI: 10.18276/ cej.2019.1-03. 